\title{
A Survey of Cheese from Small-Scale Artisanal Producers in Western Cape, South Africa
}

\author{
F. Nyamakwere, ${ }^{1,2}$ G. Esposito, ${ }^{1,3}$ K. Dzama, ${ }^{1}$ M. Muller, ${ }^{4}$ E. I. Moelich $\left(\mathbb{D},{ }^{4}\right.$ \\ and E. Raffrenato $\mathbb{D}^{1,3}$ \\ ${ }^{1}$ Department of Animal Sciences, Stellenbosch University, Private Bag X1, Matieland 7602, Stellenbosch, South Africa \\ ${ }^{2}$ Department of Animal Sciences, University of Eswatini, Luyengo Campus, Luyengo M205, Kwaluseni, Eswatini \\ ${ }^{3} R U M ぬ N$ Consulting, Reggio Emilia, Italy \\ ${ }^{4}$ Department of Food Science, Stellenbosch University, Private Bag X1, Matieland 7602, Stellenbosch, South Africa
}

Correspondence should be addressed to E. Raffrenato; emiliano@sun.ac.za

Received 23 August 2021; Accepted 7 October 2021; Published 29 October 2021

Academic Editor: Alessandro Di Cerbo

Copyright (c) 2021 F. Nyamakwere et al. This is an open access article distributed under the Creative Commons Attribution License, which permits unrestricted use, distribution, and reproduction in any medium, provided the original work is properly cited.

\begin{abstract}
The objective of this study was to assess cow milk cheese from small-scale producers in the Western Cape Province, South Africa. Forty cheeses were selected, and microbiological data of the samples were analyzed with age (1, 3, 6 months), cheese type (Cheddar, Gouda, other), and milk type (raw and pasteurized) as main factors. Rapid sensory characterization was done using the sorting technique. The results indicated that the available cheese types were diverse, with minority (20.0\%) in the Gouda group followed by Cheddar (32.5\%) and "other" (47.5\%). Most of these cheeses (45\%) were aged three months and produced using pasteurized milk (92.5\%). Five cheese samples did not meet the European Union standards for coliforms, and four samples tested positive for Escherichia coli. Sensory analysis results showed a clear differentiation between mature and young cheese. In conclusion, the current market presents more diverse milder cheeses, and there is a need to follow proper hygiene protocols to reduce cross-contamination.
\end{abstract}

\section{Introduction}

The South African dairy industry has shown an upward growth both in production and in consumption of cheese mainly produced with cow's milk, with per capita consumption reported to have increased by approximately $18.5 \%$ since 1995 [1]. This growth has been attributed to different factors such as population growth, dynamic economic developments, urbanization, income increase, and changing eating habits and lifestyle [2, 3]. Within South Africa (SA), the Western Cape Province (WC) has shown much growth, since most of the dominating milk and cheese producers are situated in this province [4]. The growth in consumption has also resulted in the growth of several small-scale cheese producers in the country with most of the cheese produced having English, Italian, Dutch, French, or Sweden origins and so do most of its producers $[5,6]$.
Many studies, especially those conducted in Europe, have proved that cheese from small-scale producers, in particular artisanal cheese, has unique favorable characteristics driven mostly by a range of factors, such as the climate, animal feed, milk type, and processing techniques $[7,8]$. In addition, the host-associated microbiota of raw milk is believed to be partially responsible for the distinctive aroma, texture, and taste that only cheese produced from raw milk can offer $[9,10]$. These unique characteristics make cheese varieties different from each other, and thus they allow access to different niche markets. However, compared to European regions, South Africa has few small-scale cheese producers with cheese production being more at a commercial level. According to the available literature, scientific studies on available SA cheese are scarce.

This study, therefore, aimed at assessing the available semihard and hard cheese from small-scale producers in the 
Western Cape, SA, being the most representative province in the country for both market and cheese production. The study provides an overview of the cheese available on the market with reference to its respective specific sensory and microbiological quality. The current study of small-scale cheese producers of the WC can assist farmers in other regions to understand the artisanal markets. Those smallscale farmers could also use these results to improve their allyear sustainability, diversify their offer to the market, and ultimately increase their profitability.

\section{Materials and Methods}

Cow milk cheese producers in South Africa were identified using Google maps, snowball technique, South African Dairy Societies databases, and information from Mulder and Wasserfall [6]. The producers were classified according to their daily cheese production as large (producing more than $1000 \mathrm{~kg}$ of cheese/day), medium (producing between 1000 and $300 \mathrm{~kg} /$ day), or small (producing less than $300 \mathrm{~kg} /$ day). Small-scale producers were then further classified as those producers manufacturing $<300 \mathrm{~kg}$ of cheese per day without mechanized equipment and/or those producing more natural specialty cheese types. A total of 68 cheese producers (large, medium, and small) were identified across South Africa, and the majority (38 producers) were in the Western Cape Province; this province was therefore selected for the study.

2.1. Cheese Sample Collection. A total of 40 cheeses were collected in November 2016 from small-scale producers, over a period of two weeks. All the available cheeses were selected without focusing on their specific characteristics. The identified farms or factories were visited, and producers were asked open questions to gather information regarding animal feed, milk type, milk source, cheese-making ingredients, use of growth hormones, and antibiotics (Table 1). Respondents were given an overview of the study's aim and were assured that their responses were confidential.

The majority of the samples $(n=29)$ were collected directly from the respective production sites and a few $(n=11)$ from specialty shops or larger retail outlets. Information such as the expiring dates and ingredients list was noted from the labels. All selected samples were within the stipulated "use by" or "best before" date. The samples were also olfactorily and visually assessed by the researcher, to note any unfavorable aromas and appearances. The cheeses were classified based on type (Gouda, Cheddar, and other), age (1, 3 , and 6 months), and milk type (raw or pasteurized). The "other" group consisted of cheese types such as Pecorino, Gruyere, Grana, Caciotta, Asiago, Colby, Danbo, Havarti, Raclette, Montasio, Kashkaval, Fontina, and Maasdam. The latter group also included other cheeses with unique local names and origins such as the Stanford, Boland, and $\mathrm{Hu}$ guenot. Samples $(200 \mathrm{~g})$ were collected in duplicate from each cheese type, kept at $4^{\circ} \mathrm{C}$ in their original packaging during transportation to the laboratory, and stored until analysis. Sensory and microbial analysis were performed within a week from collection.
TABLE 1: Questions posed to the producers.

Do you have your own farm?

Do your cows graze on natural pastures?

Do you use antibiotics or artificial growth hormones?

Do you use raw milk for cheese making? If yes, why do you prefer using raw milk?

Do you use $100 \%$ cow milk?

How much cheese do you produce per day?

How many types of cheeses do you produce?

List all the ingredients that you use for cheese processing.

Do you use animal rennet?

2.2. Microbiology Analysis. Microbiological analyses were done according to the Official Methods of Analysis of AOAC International [11]. Briefly, cheese samples were weighed $(10 \mathrm{~g})$ into sterile stomacher bags, and then $9 \mathrm{ml}$ of ringer solution was added and homogenized for 2 minutes with a bag mixer. After homogenization, $1 \mathrm{ml}$ of the content was extracted from the bags for up to three-fold serial dilutions for aerobic colony counts (ACCs) and two-fold serial dilutions for coliforms and Escherichia coli counts. Aerobic colony counts (ACCs) were determined by plating samples on Petrifilm Aerobic Count Plate (3M, Johannesburg, South Africa). Coliforms and E. coli counts were enumerated by plating samples on Petrifilm E. coli/Coliform Count Plate (3M, Johannesburg, South Africa). All plates were incubated at $32^{\circ} \mathrm{C} \pm 1^{\circ} \mathrm{C}$ for 48 hours.

Listeria monocytogenes was isolated using the rapid L. monocytogenes plates (Agar/Gelose) with the in vitro test (Bio-Rad, France). Briefly, 57.4 g of the Fraser agar powder was diluted with distilled water in a one litre bottle and mixed to dissolve. The solution was autoclaved for 15 minutes at $121^{\circ} \mathrm{C}$ and allowed to cool to below $50^{\circ} \mathrm{C}$. One vial of SR0156E (Half Fraser Selective Supplement, Bio-Rad, France) was added to the autoclaved solution, and then $90 \mathrm{ml}$ of the agar solution was poured into the frozen-thawed cheese blocks $(10 \mathrm{~g})$ and homogenized for 120 seconds. The homogenate was incubated for 24 hours at $30^{\circ} \mathrm{C}$ and then plated on the L. monocytogenes plates. Plates were incubated for 24 hours at $37^{\circ} \mathrm{C}$. Bacterial counts were converted to logarithm to the base 10 for statistical analysis.

2.3. Sensory Analysis. Rapid sensory characterization was conducted using the sorting technique followed by a descriptive step. Sorting is particularly suitable when the number of stimuli to be investigated is large and assessors are asked to sort together stimuli based on their perceived similarities [12]. The existing panel of 16 trained sensory assessors (all female between the ages of 40 and 65) with several years of experience in descriptive sensory analysis was used. Panel members were selected based on their availability for the duration of the project as well as good sensory acuity when performing a screening test for basic tastes (sweet, sour, salty, and bitter) [13]. The 40 cheeses were divided into two major groups, so as to only serve a smaller sample set to assessors per session to reduce assessor fatigue [14], and evaluation was done on two consecutive days. The first group consisted of all Cheddar- and Gouda-like cheese 
types, while the second group consisted of all the cheeses included in the "other" group.

Three rind-free cubes $(1 \mathrm{~cm} \times 1 \mathrm{~cm} \times 1 \mathrm{~cm})$ of fresh cheese were evaluated for each sample after one hour of warming to room temperature $\left(21^{\circ} \mathrm{C}\right)$. Samples were served in sealed plastic cups coded by random three-digit codes, and order of sample presentation was randomized across assessors. Unsalted crackers, water, and dried apples were available for palate cleansing between samples. The assessors were asked to focus mainly on texture and flavor attributes to sort the cheeses. They were allowed to have not more than six groups and to put as many cheeses as they wanted in each group. There was no time limit on the individuals' performance of the task. Following the sorting task, they briefly described each group with one or a few descriptors from the provided list of attributes (Table 2). The list of texture and flavor attributes was compiled by the researcher according to Muir et al. [15] and Murray and Delahunty [16]. Sensory analysis of samples was conducted under standard lighting and controlled temperature $\left(21^{\circ} \mathrm{C}\right)$ conditions in a sensory laboratory fitted with individual tasting booths.

2.4. Statistical Analysis. Forty artisanal cheeses were classified based on cheese type (Cheddar, Gouda, other), age (1, 3, 6 months), and milk type (raw and pasteurized). Microbial data was analyzed using the GLIMMIX procedures of SAS [SAS v.9.3 (Statistical Analysis System, SAS Institute (2012), Inc., Cary, NC)]. Cheese type, age, and milk type were treated as fixed factors, and place of origin was treated as a random factor. The Kenward-Roger estimation for degrees of freedom was done for the unbalanced groups of samples. For comparison, groups were weighted for the inverse of the estimated variance, and no differences were noted. The Tukey-Kramer test was used to determine the differences between data means at $5 \%$ significance level. Least squares means comparison was done using groupings.

Sorting data was subjected to DISTATIS analysis [12], to derive a compromise spatial representation of the consensus (dis) similarity of the cheeses. A contingency table was constructed to analyze descriptors associated with the cheese samples, and the list of descriptors was reduced by discarding descriptors used by few $(10 \%)$ of the assessors. The contingency table was submitted to a correspondence analysis (CA), to visualize the relationship between cheese type and descriptor counts. Cheeses were also grouped using the cluster analysis on the $n$-dimensional Euclidean distances derived from the full compromise dissimilarity matrix from DISTATIS using Ward's method. All analyses were done using the Statistica software version 12 (StatSoft Inc., 2013).

\section{Results and Discussion}

A total of 40 cheese samples were obtained from cheese factories $(n=29)$ and retail specialty stores $(n=11$; Table 3$)$. Raw milk cheese $(7.5 \%, n=3)$ accounted for a very small part of the small-scale cheese market. Similar results were reported in a survey by Brooks et al. [17] in the United States of
TABLE 2: List of flavor and texture attributes used for the sorting tasks.

\begin{tabular}{lc}
\hline Flavor attributes & Texture attributes \\
\hline Sour taste & Adhesive/sticky \\
Aftertaste & Creamy \\
Bitter taste & Crumbly \\
Buttery & Dry/grainy \\
Caramel & Firmness on palate \\
Cowy & Moist \\
Creamy & \\
Dairy/fermented sour & \\
Earthy & \\
Fruity & \\
Moldy & \\
Mushroom & \\
Nutty & \\
Rancid & \\
Salty taste & \\
Savory & \\
Smokey & \\
Soapy/chemical & \\
Spicy & \\
Sweet taste & \\
Yeast &
\end{tabular}

The list was adapted and modified from Muir et al. [15] and Murray and Delahuntry [16].

America. This could be because of increased concerns over the safety of raw milk cheese due to the potential ability of food-borne pathogens to survive the manufacturing and aging processes. In addition, other reported benefits of using pasteurized compared to raw milk include production of cheese with a greater consistency in terms of quality [18]. This is due to the fact that all the acid development is mainly from the added starter culture; thus, the rate and extent of acidification can be controlled resulting in more consistent products [18].

The results further indicated that the Western Cape artisan cheese market is diverse with majority of the cheese collected falling into the "other" group $(47.5 \%, n=19)$ followed by Cheddar (32.5\%, $n=13)$ and Gouda (20.0\%, $n=8$ ). These results agree with those of Ronquest-Ross et al. [1] who reported that Cheddar and Gouda are among the top cheese types consumed in South Africa. Most $(45 \%, n=22)$ of the collected cheeses were three months old, whereas 35\% $(n=8)$ were six months old and 20\% $(n=10)$ were one month old. This suggests that the current market presents milder (three months) compared to mature cheese. Further studies should test if this is in line with market preferences.

All the identified and collected cheeses were reported to be manufactured using non-animal rennet. According to the producers, this is mainly due to the sensitivity of the vegetarian and vegan communities in the South African markets. Producers also aim to produce more natural cheese by ensuring they use milk from free-ranging grazing cows without any history of growth hormone use and minimize use of artificial ingredients. Sixty-nine percent of the producers indicated that they used milk from their own farms with cows on pasture-based diets. Additionally, most of the producers $(60 \%)$ also indicated that they did not use any 
TABLE 3: Cheese samples from small-scale producers, classified per cheese type, cow breed, milk type, and aging period.

\begin{tabular}{|c|c|c|c|c|c|c|}
\hline Cheese type & Cheese code & Cow breed & Milk type & Feed type & Milk source & Aging period \\
\hline Asiago (As) & AsS1P & Mixed $^{1}$ & Pasteurized & Pasture $^{2}$ & Buying & 3 \\
\hline Caciotta $(\mathrm{Ca})$ & CaS1P & Mixed $^{1}$ & Pasteurized & Pasture $^{2}$ & Buying & 3 \\
\hline Cheddar (Ch) & ChS2R & Mixed $^{1}$ & Raw & Pasture $^{2}$ & Own animals & 6 \\
\hline Cheddar (Ch) & ChS3R & Guernsey & Raw & Pasture $^{2}$ & Own animals & 6 \\
\hline Cheddar (Ch) & ChS3R & Guernsey & Raw & Pasture $^{2}$ & Own animals & 3 \\
\hline Cheddar (Ch) & ChS4P & - & Pasteurized & - & Own animals & 1 \\
\hline Cheddar (Ch) & ChS5P & Mixed $^{1}$ & Pasteurized & $\mathrm{TMR}^{3}$ & Own animals & 1 \\
\hline Cheddar (Ch) & ChS5P & Mixed $^{1}$ & Pasteurized & $\mathrm{TMR}^{3}$ & Own animals & 3 \\
\hline Cheddar (Ch) & ChS6P & Jersey & Pasteurized & Pasture $^{2}$ & Own animals & 3 \\
\hline Cheddar (Ch) & ChS6P & Jersey & Pasteurized & Pasture $^{2}$ & Own animals & 6 \\
\hline Cheddar (Ch) & ChS7P & Mixed $^{1}$ & Pasteurized & Pasture $^{2}$ & Buying & 3 \\
\hline Cheddar (Ch) & ChS8P & Mixed $^{1}$ & Pasteurized & $\mathrm{TMR}^{3}$ & Own animals & 1 \\
\hline Cheddar (Ch) & ChS9P & Mixed $^{1}$ & Pasteurized & $\mathrm{TMR}^{3}$ & Own animals & 3 \\
\hline Cheddar (Ch) & ChS9P & Mixed $^{1}$ & Pasteurized & $\mathrm{TMR}^{3}$ & Own animals & 3 \\
\hline Cheddar (Ch) & ChS9P & Jersey & Pasteurized & Pasture $^{2}$ & Own animals & 3 \\
\hline Colby (Co) & CoS10P & Jersey \& Holstein & Pasteurized & Pasture $^{2}$ & Both $^{4}$ & 1 \\
\hline Danbo (Da) & DaS10P & Jersey \& Holstein & Pasteurized & Pasture $^{2}$ & Both $^{4}$ & 1 \\
\hline Fontina (Fo) & FoS1P & Mixed $^{1}$ & Pasteurized & Pasture $^{2}$ & Buying & 3 \\
\hline Gouda (Go) & GoS10P & Jersey \& Holstein & Pasteurized & Pasture $^{2}$ & Both $^{4}$ & 3 \\
\hline Gouda (Go) & GoS6P & Jersey & Pasteurized & Pasture $^{2}$ & Own animals & 3 \\
\hline Gouda (Go) & GoS5P & Mixed $^{1}$ & Pasteurized & $\mathrm{TMR}^{3}$ & Own animals & 1 \\
\hline Gouda (Go) & GoS5P & Mixed $^{1}$ & Pasteurized & $\mathrm{TMR}^{3}$ & Own animals & 3 \\
\hline Gouda (Go) & GoS11P & Mixed $^{1}$ & Pasteurized & Pasture $^{2}$ & Buying & 3 \\
\hline Gouda (Go) & GoS4P & Jersey \& Holstein & Pasteurized & Pasture $^{2}$ & Own animals & 1 \\
\hline Gouda (Go) & GoS12P & Mixed $^{1}$ & Pasteurized & Pasture $^{2}$ & Own animals & 3 \\
\hline Gouda (Go) & GoS13P & Mixed $^{1}$ & Pasteurized & Pasture $^{2}$ & Own animals & 3 \\
\hline Grana (Ga) & GaS10P & Jersey \& Holstein & Pasteurized & Pasture $^{2}$ & Both $^{4}$ & 6 \\
\hline Gruyere (Gr) & GrS4P & - & Pasteurized & - & Own animals & 1 \\
\hline Gruyere (Gr) & GrS10P & Jersey \& Holstein & Pasteurized & Pasture $^{2}$ & Both $^{4}$ & 6 \\
\hline Havarti (Ha) & HaS10P & Jersey \& Holstein & Pasteurized & Pasture $^{2}$ & Both $^{4}$ & 3 \\
\hline Huguenot $(\mathrm{Hu})$ & HuS14P & Jersey & Pasteurized & Pasture $^{2}$ & Own animals & 6 \\
\hline Kashkaval (Ka) & KaS11P & Mixed $^{1}$ & Pasteurized & Pasture $^{2}$ & Buying & 3 \\
\hline Maasdam (Ma) & MaS5P & Mixed $^{1}$ & Pasteurized & Pasture $^{2}$ & Own animals & 1 \\
\hline Montasio (Mo) & MoS1P & Mixed $^{1}$ & Pasteurized & Pasture $^{2}$ & Buying & 6 \\
\hline Pecorino $(\mathrm{Pe})$ & PeS15P & Jersey & Pasteurized & Pasture $^{2}$ & Both $^{4}$ & 3 \\
\hline Pecorino (Pe) & PeS5P & Mixed $^{1}$ & Pasteurized & $\mathrm{TMR}^{3}$ & Own animals & 3 \\
\hline Port Salut (Po) & PoS14P & Jersey & Pasteurized & Pasture $^{2}$ & Own animals & 3 \\
\hline Raclette $(\mathrm{Ra})$ & RaS10P & Jersey \& Holstein & Pasteurized & Pasture $^{2}$ & Both $^{4}$ & 6 \\
\hline Royal Ashton (Ro) & RoS5P & Mixed $^{1}$ & Pasteurized & $\mathrm{TMR}^{3}$ & Own animals & 1 \\
\hline Stanford $(\mathrm{St})$ & StS10P & Jersey \& Holstein & Pasteurized & Pasture $^{2}$ & Both $^{4}$ & 3 \\
\hline
\end{tabular}

${ }^{1}$ Mixed breed, i.e., Jersey, Holstein-Friesian, and crosses of both, ${ }^{2}$ pasture-based with few supplements, ${ }^{3}$ total mixed rations, ${ }^{4}$ buying milk and also having own animals, - : some producers were not sure about their milk origins resulting in the missing data. For cheese codes the $1^{\text {st }} 2$ letters refer to the cheese type, S1 to S15 refer to the supplier, and P (pasteurized) and R (raw) refer to the milk type.

preservatives or artificial colorants during manufacture. Six of the sampled cheeses were also among the top South African award-winning cheeses at an international level. These cheeses are reported to have different unique characteristics, which could be attributed to different factors such as unique processing techniques, feed type, milk quality, and cow breeds $[7,8,19]$.

3.1. Microbiological Quality. Microbiological quality assessment of artisanal cheeses from small-scale producers helps to note the potential risk the products might present to the consumers. The results can also be considered when structuring public policies that aim to protect the consumer's health. Although the Cheddar $\left(1.08 \log _{10} \mathrm{CFU} / \mathrm{g}\right)$ and Gouda $\left(1.21 \log _{10} \mathrm{CFU} / \mathrm{g}\right)$ had higher $(P<0.05)$ coliform counts compared to "other" cheese $\left(0.5 \log _{10} \mathrm{CFU} / \mathrm{g}\right)$ group (Figure 1), the values were still within the international limits $\left(<4.0 \log _{10} \mathrm{CFU} / \mathrm{g}\right)$ as set by the European Union (EU; Commission Regulation No. 2073/2005) [20]. On the other hand, higher $(P<0.05)$ ACCs were noted in the Cheddar (4.88 $\log _{10} \mathrm{CFU} / \mathrm{g}$ ) and "other" (4.61 $\log _{10} \mathrm{CFU} / \mathrm{g}$ ) groups compared to the Gouda (4.02 $\log _{10} \mathrm{CFU} / \mathrm{g}$ ) cheese. The ACC values were below the recommended EU limits for raw milk cheese of $7.0 \log _{10} \mathrm{CFU} / \mathrm{g}$. Overall, the Cheddar type was noted to have more microbial counts compared to Gouda and "other" groups. This can be a result of higher moisture content observed for this cheese type. In fact, cheese with higher moisture content has higher water activity values, which favors microbial growth [21]. 


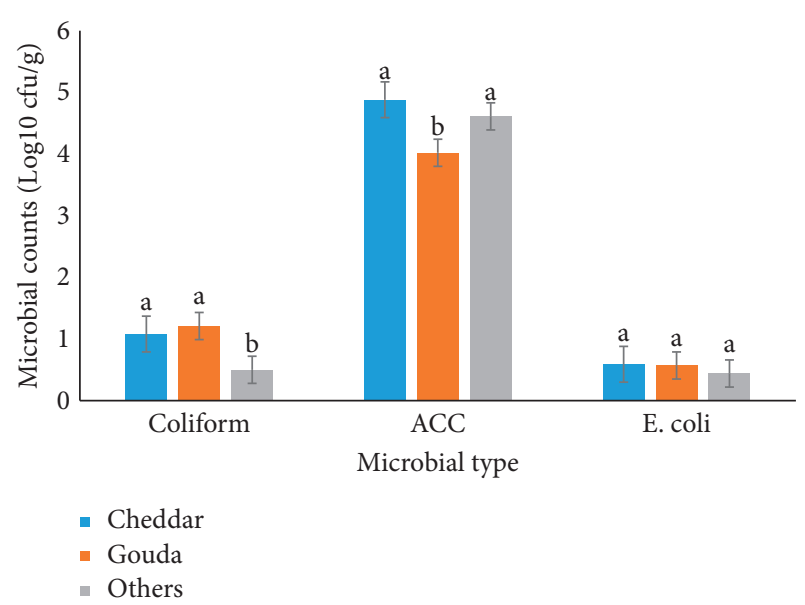

Figure 1: Microbial counts according to cheeses type; for each microbial type, bars with different superscripts are significantly different $(P<0.05)$. ACC: aerobic colony count; E. coli: Escherichia coli.

Microbial counts were observed to decrease with age for all cheese types. Aerobic colony counts (ACCs) were significantly higher $(P<0.05)$ in the one-month-old cheese group (5.0 $\log _{10} \mathrm{CFU} / \mathrm{g}$ ) compared to three- and six-monthold groups (Figure 2). Bacterial counts decrease as the $\mathrm{pH}$ decreases during aging with the increase of lactic acid percentage [22].

Smith et al. [23] reported ready-to-eat meat products such as polony as a source of listeriosis in SA. The country experienced one of the worst world listeriosis outbreaks in 2018. In the United States [24], it was observed that Hispanic-style cheese was a source of $L$. monocytogenes. Conversely, for this study, no samples were found to be positive for L. monocytogenes.

Overall, five samples did not fall within the EU international limits for coliform counts, and four samples had E. coli counts greater than or equal to $3 \log _{10} \mathrm{CFU} / \mathrm{g}$ which exceeded the threshold limit for dairy products. A similar survey was conducted in Belgium, and E. coli was counted in $26.7 \%$ of the samples [25]. These results are in line with other findings in the United States of America [17] and in Egypt [26], where E. coli was detected in $15 \%$ and $21.7 \%$ of the artisanal cheeses, respectively. These studies noted that E. coli positive samples were mainly from raw milk cheese. Interestingly, the current study noted that pasteurized milk cheese had higher $(P<0.05)$ coliform $\left(1.44 \log _{10} \mathrm{CFU} / \mathrm{g}\right.$; Figure 3) compared to raw milk. Therefore, pasteurization does not guarantee cheese safety, although it could do if contamination of the cheese after pasteurization is avoided [18]. Opportunities for postpasteurization contamination may occur during either processing, aging, storage, or packaging, putting the cheese at risk of contamination [25].

In this study, few producers (7.5\%) stated that they use raw milk or add raw milk to pasteurized milk during cheese processing as they considered it essential for good texture and flavor development. These favorable characteristics come as a result of proteolysis and lipolysis processes by raw milk natural microflora and enzymes [27]. However,

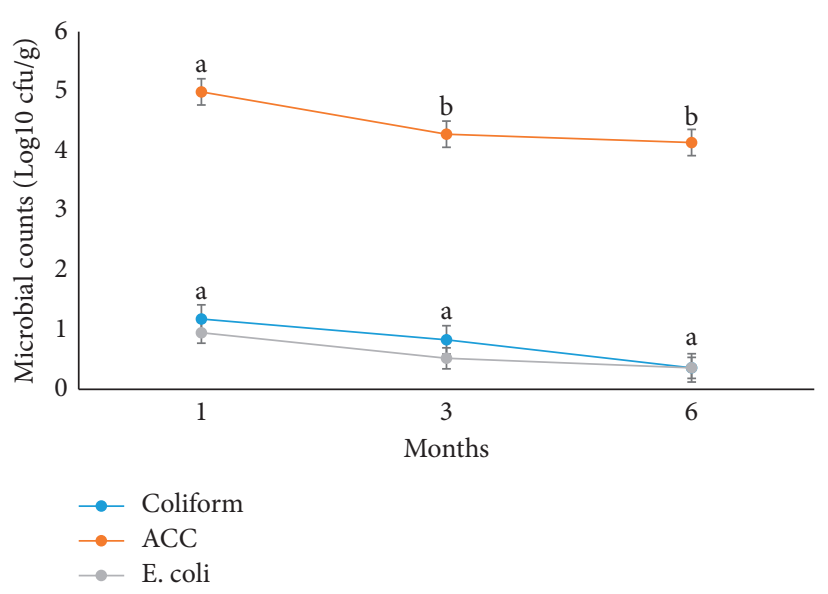

Figure 2: Microbial counts according to aging months. Different superscripts indicate a significant difference across months $(P<0.05)$. ACC: aerobic colony count; E. coli: Escherichia coli.

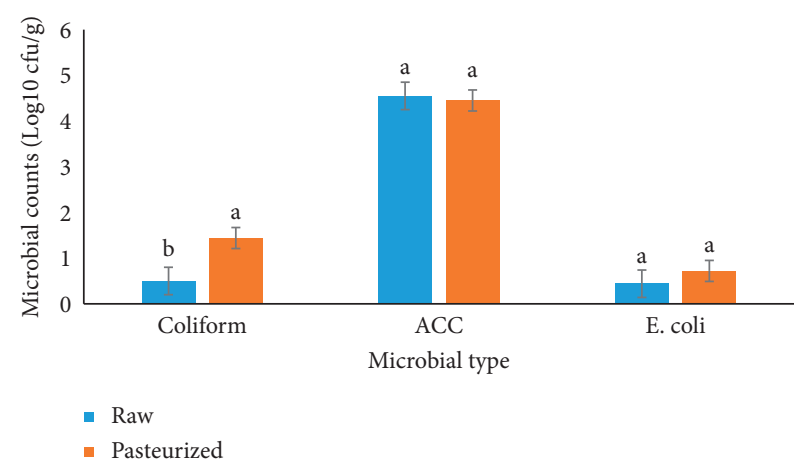

FIgURE 3: Microbial counts according to milk type. Different superscripts indicate a significant difference according to milk type $(P<0.05)$. ACC: aerobic colony count; E coli: Escherichia coli.

assessment of the microbiological quality of raw milk cheese has shown varying levels of quality and safety [17]. There are debates over the use of raw milk for cheese processing, and it is worth reviewing as consumers at times are reported to make purchasing decisions mostly based on taste, and not attitude towards safety [28].

In South Africa, raw milk can be used in cheese making, and the products should be clearly labeled to inform the consumers [29]. However, further investigation of the cheese processing plants is warranted, as the results obtained during the current study indicate that contamination may be due to poor processing and hygiene practice in the processing plants. Most artisanal cheese producers in Mexico lack enough knowledge about food safety and reported higher levels of coliforms and E. coli counts [30]. Further studies should be conducted to provide clarity regarding the actual sources of contamination and give room for implementation of appropriate control measures.

3.2. Sensory Properties. The cheese samples were divided into two groups, and results will be presented per group using the codes defined in Table 3. On the CA plot of 
Cheddar-Gouda group, the first dimension accounts for $66.72 \%$ of the explained variance, distinguishing a group of six-month-old Cheddar cheeses (ChS3R, ChS2R, and ChS6P; Figure 4) towards the left of dimension 1. These cheeses were defined more by texture attributes such as "crumbly," "dry," "hard," "grainy," and "rancid." On the right side of dimension 1, we have the one- and threemonth-old cheeses (ChS9P, ChS3R, ChS9P, ChS5P, GoS13P, GoS11P, GoS5P, ChS8P, ChS5P, GoS5P), associated with "soft on palate," "moist," "sweet," "buttery," "creamy," "sticky," and "rubbery". Dimension 2 could not clearly distinguish the Cheddar-Gouda group, and separation was slightly driven by GoS6P which was "bitter" and "earthy."

On the DISTATIS plot of the Cheddar-Gouda group, the first two principal components (PC) of the sorting matrix account for $30.1 \%$ of the total variation (Figure 5), and the remaining components each account for less than $10 \%$ of variation. Despite this relatively low proportion of explained variance, the first two dimensions are each interpretable and appear to define the structure of the product space. The first dimension clearly separates six-month-old Cheddar cheeses (ChS2R, ChS3R, and ChS6P) towards the upper left quadrant of the plot from the three-month-old cheeses, mostly Gouda (GoS5P, GoS12P, GoS13P, ChS9P, and GoS11P). Principal component 2 separates the GoS6P, GoS4P, ChS4P, ChS5P, and ChS3R cheeses from the rest of the samples. Similar distinctive groups were also noted on the cluster analysis plot (Figure 6). If we draw a vertical line at 0.8 linkage distance, three clusters can be seen, clearly grouping the cheese based on their age groups. As demonstrated by [31], clusters of the subjects can be obtained by cutting the resulting dendrogram at a particular level. The groupings for this study seem to be driven more by attributes associated with cheese maturity, which has been described as one of the most important attributes driving consumer eating quality perceptions $[16,32]$. In addition, cheese from the same producers (ChS5P and GoS5P; ChS4P and ChS4P) were noted to be placed in the same groups on the DISTATIS plot (Figure 5). This suggests that cheese from the same producers have similar flavor and texture properties. This might be a result of similar processing techniques. Raw milk cheese (ChS2R and ChS3R) fell into the same groups, suggesting similar sensory characteristics (Figure 6). This is in line with findings by Van Leuven et al. [33], who observed a clear distinction between raw and pasteurized milk cheese. Flavor development during cheese ripening is influenced by the type of milk (raw vs. pasteurized) used [33].

The first dimension on the CA plot derived from the sorting data of the "other" group accounted for $58.54 \%$ of the explained variance, clearly separating RaS10P, HaS10P, and DaS10P situated towards the right of dimension 1 from the rest of the samples (Figure 7). The latter cheeses are associated with attributes such as "soft on palate," "creamy," "buttery," and "moist." Cheese samples towards the left side of dimension one, namely, PeS5P, CaS1P, GrS4P, MoS1P, GrS10P, GaS10P, and HuS14P, are associated with attributes indicating maturity, "firm on palate," "nutty," "dry," "savory," "bitter," "hard," and "fermented sour."

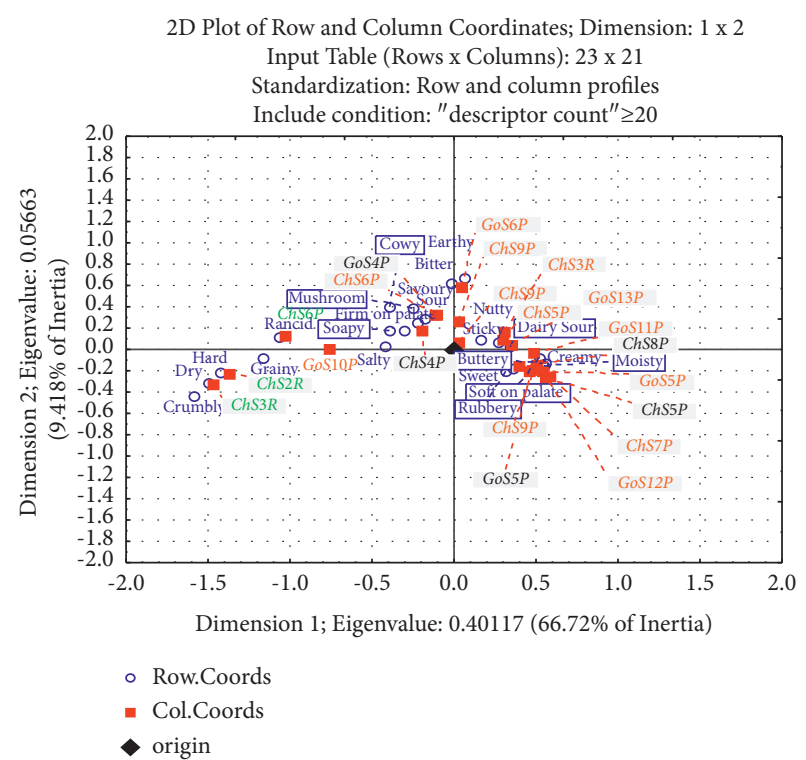

FIgURE 4: Representation of samples with texture and flavor sensory attributes in the first two dimensions of the CA plot performed on data from sorting of the "Cheddar-Gouda" group $(n=21)$. For cheese codes, the Ch refers to Cheddar and Go refers to Gouda, S1 to S15 refer to the supplier, and P (pasteurized) and R (raw) refer to the milk type. Color codes: black: 1 month; orange: 3 months; green: 6 months.

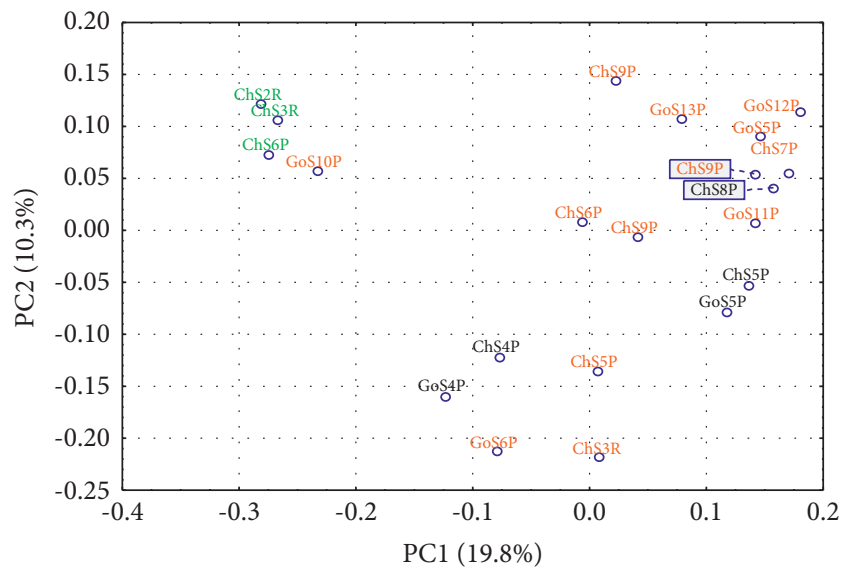

FIgURE 5: DISTATIS plot of the Cheddar-Gouda group $(n=21)$ derived from the sorting data. For cheese codes, the $\mathrm{Ch}$ refers to Cheddar and Go refers to Gouda, S1 to S15 refer to the supplier, and $\mathrm{P}$ (pasteurized) and $\mathrm{R}$ (raw) refer to the milk type. Color codes: black: 1 month; orange: 3 months; green: 6 months.

Dimension 2 showed differences in the sensory characteristics between cheese varieties with a "rubbery," "sweet," and "medium flavor" towards the positive end. The cheese samples associated with these attributes are CoS10P, $\mathrm{KaS11P}, \mathrm{StS10P}$, and PeS15P. Cheeses towards the negative side of dimension 2, namely, GaS10P, AsS1P, PoS14P, and $\mathrm{DaS10P}$, are associated with attributes such as "fermented sour," "mature," "grainy," and "sticky" texture. Closer inspection of the cluster analysis plot shows that one- and three-month-old cheeses such as the PeS5P, PeS15P, 


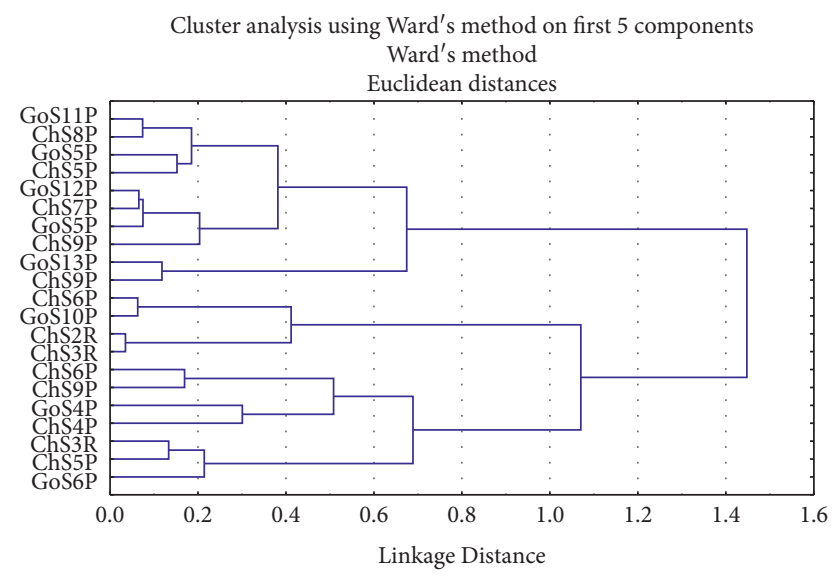

FIgURE 6: Cluster analysis of the Cheddar-Gouda group $(n=21)$ derived from the sorting data. For cheese codes, the Ch refers to Cheddar and Go refers to Gouda, S1 to S15 refer to the supplier, and $\mathrm{P}$ (pasteurized) and $\mathrm{R}$ (raw) refer to the milk type.

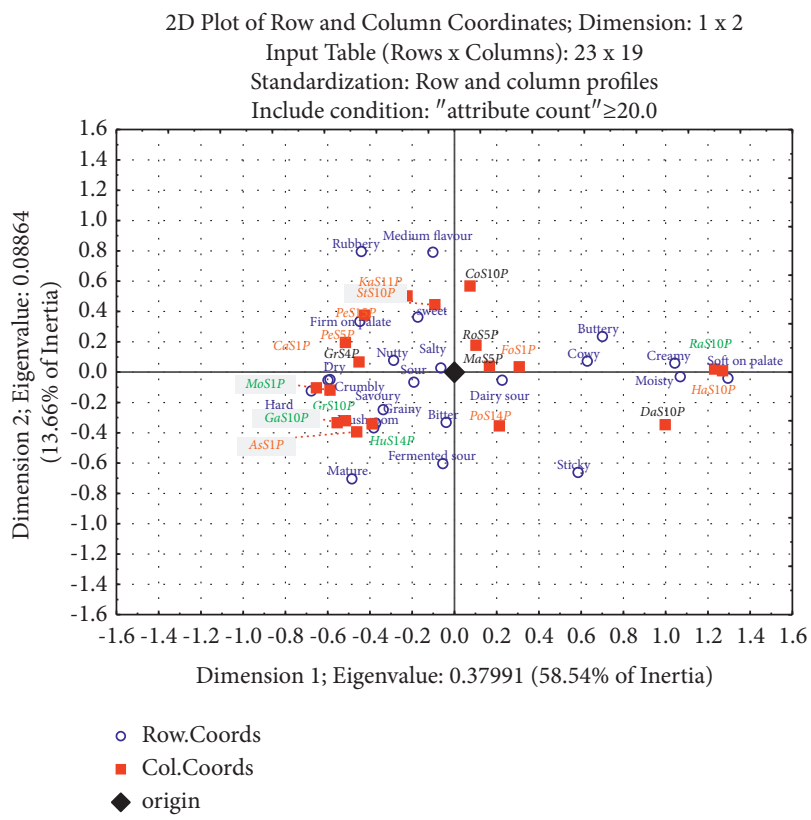

FIGURE 7: Representation of samples with texture and flavor sensory attributes in the first two dimensions of the CA plot performed on data from sorting of the "other" group $(n=19)$. For cheese codes, the $1^{\text {st }} 2$ letters refer to the cheese type, S1 to S15 refer to the supplier, and $\mathrm{P}$ (pasteurized) and $\mathrm{R}$ (raw) refer to the milk type. Color codes: black: 1 month; orange: 3 months; green: 6 months.

CoS10P, and StS10P are similar, and they are different from DaS10P, HaS10P, and RaS10P (Figure 8).

The first two PCs of the DISTATIS plot of the "other" group account for $32.8 \%$ of the total variation in the data, with PC1 clearly distinguishing DaS10P, HaS10P, and RaS10P towards the right side from the rest of the samples (Figure 9). The second PC groups the cheeses mainly according to age, separating the six-month-old GaS10P, MoS1P, GrS10P, and HuS14P (in the positive direction)

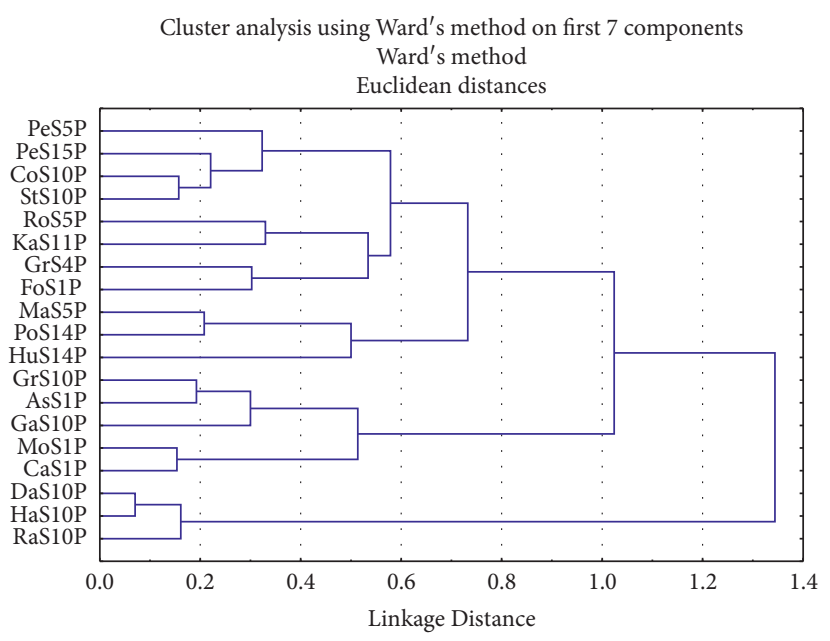

FIgURE 8: Cluster analysis of the "other" group $(n=19)$ derived from the sorting data. For cheese codes, the $1^{\text {st }} 2$ letters refer to the cheese type, S1 to S15 refer to the supplier, and P (pasteurized) and $\mathrm{R}$ (raw) refer to the milk type.

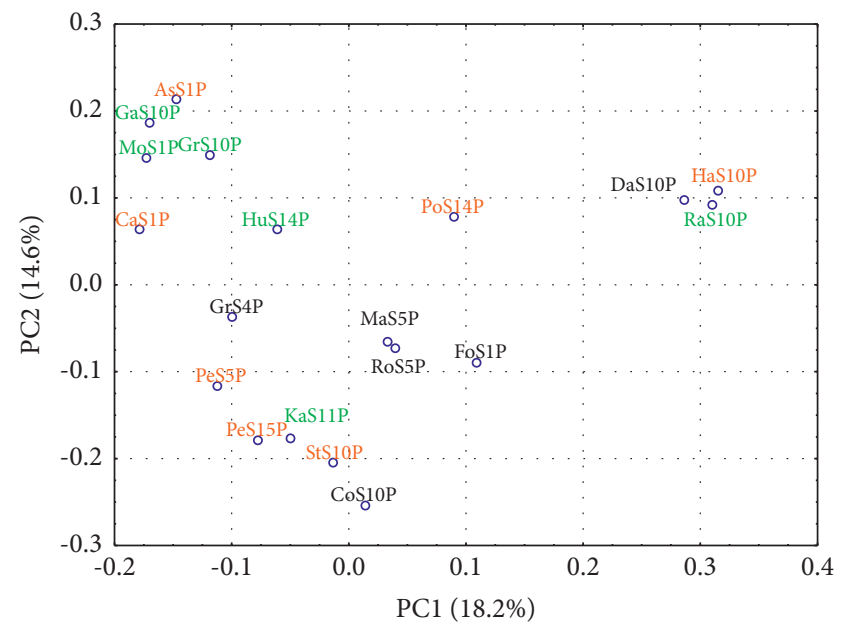

FIGURE 9: DISTATIS plot of the "other" group $(n=19)$ derived from the sorting data. For cheese codes, the $1^{\text {st }} 2$ letters refer to the cheese type, S1 to S15 refer to the supplier, and P (pasteurized) and $\mathrm{R}$ (raw) refer to the milk type. Color codes: black: 1 month; orange: 3 months; green: 6 months.

from the one- and three-month-old CoS10P, StS10P, PeS15P, KaS11P, and PeS5P (in the negative direction; Figure 9). The limited differentiation observed for the present work could be due to the fact that these cheese varieties were more closely related.

\section{Conclusion}

The results of this study indicate that most of the artisanal cheeses present in the Western Cape, South Africa, were aged for three months, which might be in line with the market demands, and that they have diverse characteristics. Producers aim at producing more natural cheese by ensuring they use milk from free-ranging grazing cows and minimize use of artificial ingredients. This study also highlighted the 
importance of following proper hygiene protocols during processing or postprocessing, thus reducing chances of cross-contamination, regardless of the milk type used. Cheese sensory differences were noted to be driven mostly by texture and flavor attributes linked to maturity. The broad microbiological and sensory description of cheese helps to give valuable information on product quality, to allow producers to expand their markets with the possibility of exporting and thereby growing internationally, protecting their niche markets.

\section{Data Availability}

The data used to support the findings of this study are available from the corresponding author upon request.

\section{Ethical Approval}

Ethics approval was not required for this research.

\section{Disclosure}

The funders (NRF and MMEG) were not involved in the study design; collection, analysis, and interpretation of data; writing of the manuscript; or decision to submit the manuscript for publication.

\section{Conflicts of Interest}

The authors declare no conflicts of interest.

\section{Acknowledgments}

Special thanks are due to the South African small-scale cheese producers who participated in this study. This research was funded by the National Research Foundation (NRF), South Africa. The authors also acknowledge the Margaret McNamara Education Grants (MMEG) for a scholarship to FN.

\section{References}

[1] L. C. Ronquest-Ross, N. Vink, and G. O. Sigge, "Food consumption changes in South Africa since 1994," South African Journal of Science, vol. 111, pp. 1-12, 2015.

[2] O. A. Ndambi and T. Hemme, "An economic comparison of typical dairy farming systems in South Africa, Morocco, Uganda and Cameroon," Tropical Animal Health and Production, vol. 41, no. 6, pp. 979-994, 2009.

[3] OECD, OECD-FAO Agricultural Outlook 2015, OECD Publishing, Paris, France, 2015.

[4] Milk South Africa, Annual Industry Information Reports, vol. 20, Milk South Africa, Pretoria, South Africa, 2017https:// www.milksa.co.za.

[5] F. Nyamakwere, G. Esposito, K. Dzama, and E. Raffrenato, "A review of artisanal cheese making: an African perspective," South African Journal of Animal Science, vol. 51, no. 3, pp. 296-309, 2021.

[6] K. Mulder and R. Wasserfall, Cheeses of South Africa: Artisanal Producers and Their Cheeses, Sunbird Publishers, Cape Town, South Africa, 2013.
[7] D. S. Home and J. M. Banks, Rennet-Induced Coagulation of Milk, Elsevier Ltd, Scotland, UK, 2004.

[8] A. Nudda, G. Battacone, O. Boaventura Neto et al., "Feeding strategies to design the fatty acid profile of sheep milk and cheese," Revista Brasileira de Zootecnia, vol. 43, no. 8, pp. 445-456, 2014.

[9] A. Terzic-Vidojevic, M. Vukasinovic, K. Veljovic, M. Ostojic, and L. Topisirovic, "Characterization of microflora in homemade semi-hard white Zlatar cheese," International Journal of Food Microbiology, vol. 114, no. 1, pp. 36-42, 2007.

[10] A. Bonanno, G. Tornambè, V. Bellina et al., "Effect of farming system and cheesemaking technology on the physicochemical characteristics, fatty acid profile, and sensory properties of Caciocavallo Palermitano cheese," Journal of Dairy Science, vol. 96, no. 1, pp. 710-724, 2013.

[11] AOAC, Official Methods of Analysis, Bacterial and E. coli/ coliform Counts in Dairy Products (Petrifilm), Method No. 986.33 and 989.10, The Association of Official Analytical Chemists, Rockville, MD, USA, 2014.

[12] H. Abdi, D. Valentin, S. Chollet, and C. Chrea, "Analyzing assessors and products in sorting tasks: DISTATIS, theory and applications," Food Quality and Preference, vol. 18, no. 4, pp. 627-640, 2007.

[13] H. T. Lawless and H. Heymann, Sensory Evaluation of Food: Principles and Practices, Springer, New York, NY, USA, 2nd edition, 2010.

[14] J. Lahne, H. Abdi, and H. Heymann, "Rapid sensory profiles with DISTATIS and barycentric text projection: an example with amari, bitter herbal liqueurs," Food Quality and Preference, vol. 66, pp. 36-43, 2018.

[15] D. D. Muir, E. A. Hunter, J. M. Banks, and D. S. Horne, "Sensory properties of hard cheese: identification of key attributes," International Dairy Journal, vol. 5, no. 2, pp. 157-177, 1995.

[16] J. M. Murray and C. M. Delahunty, "Mapping consumer preference for the sensory and packaging attributes of Cheddar cheese," Food Quality and Preference, vol. 11, no. 5, pp. 419-435, 2000.

[17] J. C. Brooks, B. Martinez, J. Stratton, A. Bianchini, R. Krokstrom, and R. Hutkins, "Survey of raw milk cheeses for microbiological quality and prevalence of foodborne pathogens," Food Microbiology, vol. 31, no. 2, pp. 154-158, 2012.

[18] M. E. Johnson, "A 100-year review: cheese production and quality," Journal of Dairy Science, vol. 100, no. 12, pp. 9952-9965, 2017.

[19] F. Serrapica, F. Masucci, A. Di Francia et al., "Seasonal variation of chemical composition, fatty acid profile, and sensory properties of a mountain pecorino cheese," Foods, vol. 9, no. 8, p. 1091, 2020.

[20] Commission Regulation of the European Committee, "Microbiological criteria for foodstuff," pp. 1-26, Official Journal of the European Union, Brussels, Belgium, 2005.

[21] A. Bellio, D. M. Bianchi, N. Vitale, L. Vernetti, S. Gallina, and L. Decastelli, "Behavior of Escherichia coli O157: H7 during the manufacture and ripening of Fontina protected designation of origin cheese," Journal of Dairy Science, vol. 101, no. 6, pp. 4962-4970, 2018.

[22] C. Chatelard-Chauvin, F. Pelissier, S. Hulin, and M. C. Montel, Behaviour of Listeria monocytogenes in raw milk Cantal type cheeses during cheese making, ripening and storage in different packaging conditions, Food Control, vol. 54, pp. 53-65, 2015.

[23] A. M. Smith, N. P. Tau, S. L. Smouse et al., Outbreak of Listeria monocytogenes in South Africa, 2017- laboratory activities and 
experiences associated with wholeenome sequencing analysis of isolates, Foodborne Pathogens and Disease, vol. 16, no. 7, pp. 524-530, 2019.

[24] Y. Chen, Y. Luo, H. Carleton et al., "Whole genome and core genome multilocus sequence typing and single nucleotide polymorphism analyses of Listeria monocytogenes isolates associated with an outbreak linked to cheese, United States," Applied and Environmental Microbiology, vol. 83, Article ID e00633-17, 2017.

[25] E. Lahou and M. Uyttendaele, "Growth potential of Listeria monocytogenes in soft, semi-soft and semi-hard artisanal cheeses after post-processing contamination in deli retail establishments," Food Control, vol. 76, pp. 13-23, 2017.

[26] R. A. Ombarak, A. Hinenoya, S. P. Awasthi et al., "Prevalence and pathogenic potential of Escherichia coli isolates from raw milk and raw milk cheese in Egypt," International Journal of Food Microbiology, vol. 221, pp. 69-76, 2016.

[27] D. K. Hickey, T. P. Guinee, J. Hou, and M. G. Wilkinson, "Effects of variation in cheese composition and maturation on water activity in Cheddar cheese during ripening," International Dairy Journal, vol. 30, no. 1, pp. 53-58, 2013.

[28] K. B. Waldman and J. M. Kerr, "Is food and drug administration policy governing artisan cheese consistent with consumers' preferences?” Food Policy, vol. 55, pp. 71-80, 2015.

[29] Government Notice, "Regulation related to milk and dairy products," pp. 1-19, 1997.

[30] A. Chávez-martínez, P. Paredes-Montoya, R. Sánchez-Vega, and E. Santellano-Estrada, "Microbial quality and prevalence of foodborne pathogens of cheeses commercialized at different retail points in Mexico," Food Science and Technology, vol. 61, pp. 1-8, 2019.

[31] P. Courcoux, P. Faye, and E. M. Qannari, "Determination of the consensus partition and cluster analysis of subjects in a free sorting task experiment," Food Quality and Preference, vol. 32, pp. 107-112, 2014.

[32] M. D. Yates and M. A. Drake, "Texture properties of gouda cheese," Journal of Sensory Studies, vol. 22, no. 5, pp. 493-506, 2007.

[33] I. Van Leuven, T. Van Caelenberg, and P. Dirinck, "Aroma characterisation of Gouda-type cheeses," International Dairy Journal, vol. 18, no. 8, pp. 790-800, 2008. 